\title{
Coupled membrane transporters reduce noise
}

\author{
Luca Cardelli and Luca Laurenti $\odot$ \\ Department of Computer Science, University of Oxford, Oxford OX1 3QD, United Kingdom \\ Attila Csikasz-Nagy $\odot$ \\ Randall Division of Cell and Molecular Biophysics and Institute of Mathematical and Molecular Biomedicine, \\ King's College London, London, United Kingdom \\ and Pázmány Péter Catholic University, Faculty of Information Technology and Bionics Budapest, Hungary
}

(Received 21 July 2019; published 27 January 2020)

\begin{abstract}
Molecular systems are inherently probabilistic and operate in a noisy environment, yet, despite all these uncertainties, molecular functions are surprisingly reliable and robust. The principles used by natural systems to deal with noise are still not well understood, especially in a nonhomogeneous environment where molecules can diffuse across different compartments. In this paper we show that membrane transport mechanisms have very effective properties of noise reduction. In particular, we show that active transport mechanisms (those that can transport against a gradient of concentration by using energy or by means of the concentration gradient of other substances), such as symporters and antiporters, have surprising efficiency in noise reduction, which outperforms passive diffusion mechanisms and are well below Poisson levels. We link our results to the coupled transport of potassium, sodium, and glucose to show that the noise in internal glucose level can be greatly reduced. Our results show that compartmentalization can be a highly effective mechanism of noise reduction and suggests that membrane transport could give this extra benefit, contributing to the emergence of complex compartmentalization in eukaryotes.
\end{abstract}

DOI: 10.1103/PhysRevE.101.012414

\section{INTRODUCTION}

Molecular processes contain an inherent element of stochasticity due to reactions involving molecules present in low numbers. Such a noise interferes with cellular functions and propagates to all dependent processes [1]. For example, noise in messenger RNA (mRNA) transcription propagates to translation processes often leading to super-Poisson variability in protein levels [2]. Hence, at a first sight, it seems impossible that complex multistep processes may exhibit highly controlled behavior. Nevertheless, biological systems perform their functions in a surprisingly reliable way. Therefore, they must incorporate mechanisms that increase robustness and reduce noise [3].

In order to understand how natural systems can reduce the noise, molecular filters have been studied [4]. Molecular filers are chemical reaction networks that are able to reduce the noise of an input molecular signal. Examples of molecular filters include feedback and feed-forward loops [5], low-pass filters [6], and annihilation filters [4]. Many theoretical studies of these systems have been performed and lower bounds on their noise-reduction capabilities have been derived $[4,7,8]$. However, the vast majority of the published works focus on noise-reduction mechanisms in a homogeneous environment

Published by the American Physical Society under the terms of the Creative Commons Attribution 4.0 International license. Further distribution of this work must maintain attribution to the author(s) and the published article's title, journal citation, and DOI. and at the single cell level, whereas much less is known about the effect that spatial compartmentalization can have on noise [9].

In this paper, we show that membrane transport mechanisms can act as efficient noise filters. Passive transport mechanisms act by diffusion in the direction of a gradient. Active transport mechanisms instead can transport materials against a gradient. This is achieved either by using energy in the form of Adenosine Triphosphate (ATP) (primary active transport) or by means of the gradient of other substances (secondary active transport). We show that active mechanisms outperform passive ones in terms of noise reduction. The observed noise reduction does not require introduction of time delays, commonly used in other noise-reducing mechanisms. In particular, we study how molecular pumps that transport two molecule types in the same direction (symporters) or the opposite directions (antiporters) can reduce internal noise in cells well below Poisson levels. Furthermore, we use the derived results to investigate how sodium-potassium pumps in combination with sodium-glucose cotransporters can filter out external molecular noise and reduce the fluctuations in intracellular glucose levels.

The idea that compartmentalization can act as a noise filter is not new [9]. However, a deep mathematical analysis is lacking and all the theoretical results are mostly limited to passive diffusive transport mechanisms $[10,11]$. Hence, our results provide a key step toward the understanding of the robustness properties of natural systems and, due to ubiquitous presence of cellular compartments in eukaryotic cells, suggest that spatial compartmentalization may be the predominant mechanism of noise reduction in eukaryotes. 

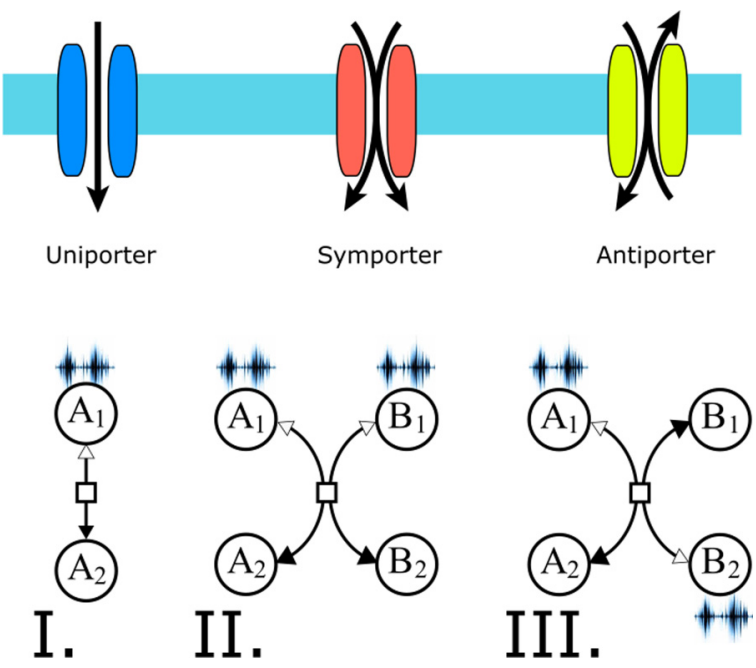

FIG. 1. Transporters: Common transmembrane transporters (top) and their respective reaction schemes [(I), (II), and (III)], where circles denote species and squares denote reversible reactions. Subscripts indicate compartment numbers (inside or outside the membrane). Direct reactions have solid arrowhead, while inverse (assumed weaker) reactions have hollow arrowheads. Ambient noise (indicated by noisy graphs) is applied to the input species.

\section{UNIPORTER}

We start our analysis by considering a simple transport mechanism between two compartments [Fig. 1(I)],

$$
A_{1} \rightarrow{ }^{k_{1}} A_{2} ; \quad A_{2} \rightarrow{ }^{k_{2}} A_{1},
$$

where molecules of species $\mathrm{A}$ are transported between two compartments and with $A_{i}$ representing the number of molecules of species A in compartment $i$. This mechanism can correspond to facilitated diffusion if the transport follows the gradient of molecules across a permeable membrane $\left(k_{1}=k_{2}\right)$ but is often used also as a simplified model of other forms of membrane transport, such as the transport of mRNA from nucleus to cytoplasm [10].

We assume molecules of A appear in compartment 1 through extracellular transport according to a noise process, which includes bursts of dimension $b_{A}>0$ and that can be modelled by the following reactions

$$
\rightarrow^{k_{A}} b_{A} A_{1} ; \quad A_{1} \rightarrow^{k_{d A}} .
$$

That is, $A_{1}$ appears in compartment 1 through a noisy process characterized by the following mean and Fano factor (ratio between variance and expectation) at steady state,

$$
E\left[A_{1}\right]_{\infty}=\frac{b_{A} k_{A}}{k_{d A}} \quad F_{A_{1}}=\frac{1+b_{A}}{2} .
$$

In this paper we use the Fano factor to quantify the noise. This is because the Fano factor allows one to compare the noise of a given species with respect to Poisson levels (Fano factor of a Poisson process is always 1 independently of the mean value). From Eq. (3) it is clear that the parameter $b_{A}$ determines the noise of $A_{1}$. In particular, for $b_{A}=1$ we have Poisson noise (Fano factor equals 1), while for $b_{A}>1$ we have super-Poisson noise.
Under this input process for $A_{1}$, we can derive the exact expressions for expectation and Fano factor of $A_{2}$ at steady state and we obtain

$$
E\left[A_{2}\right]_{\infty}=\frac{b_{A} k_{A} k_{1}}{k_{d A} k_{2}} \quad F_{A_{2}}=1+\frac{k_{1}\left(b_{A}-1\right)}{2\left(k_{1}+k_{2}+k_{d A}\right)} .
$$

The transport mechanism in Eq. (1) can filter noise when the transport is slow $\left(k_{1}\right.$ small $)$ but can never bring it below Poisson levels (Fano is lower bounded by 1). This confirms experimental observations in Ref. [12], where it is shown that slow nuclear export of transcripts may result in a reduced variability of transcripts without affecting mean abundance. Note, however, that this transport mechanism may be beneficial even when the transport is fast. In fact, when $k=k_{1}=k_{2}$ we obtain $\lim _{k \rightarrow \infty} F_{A_{2}}=\frac{3+b_{A}}{4}$, which for $b_{A}>1$ is always smaller than $F_{A_{1}}$. Note also that for the limit case $b_{A}=1$ (Poisson noise) we see that $F_{A_{2}}=1$ independently of the reaction rates.

Nevertheless, although the described mechanism can reduce noise, Eq. (4) implies that the noise reduction is inherently lower bounded by Poisson noise (Fano factor of 1). In the following sections we show that coupled transport mechanisms allow one to obtain better noise-reduction performances, and we link them to well-studied symporters and antiporters [13].

\section{SYMPORTER AND ANTIPORTER}

The first coupled active transport we consider is a symporter [Fig. 1(II)], inspired by transmembrane symporters. Membrane symporters are integral membrane proteins that are involved in the cotransport of different types of molecules across the cell membrane [13].

The symporter simultaneously transports molecules of species A and B from compartment 1 to compartment 2 and can be modelled with the following reactions:

$$
A_{1}+B_{1} \rightarrow{ }^{k_{1}} A_{2}+B_{2} ; \quad A_{2}+B_{2} \rightarrow^{k_{1} \cdot r} A_{1}+B_{1},
$$

where $A_{i}, B_{i}$ are molecules of species $\mathrm{A}$ and $\mathrm{B}$ in compartments $i \in\{1,2\}$. The above mechanism is widely used to transport one of the molecules against concentration gradients while the other follows its gradient [13]. In what follows, we show that this mechanism has also surprising properties for noise reduction. In order to illustrate this point, as for the previous case, we assume that $A_{1}$ and $B_{1}$ are subject to burst noise modelled as

$$
\rightarrow^{k_{A}} b_{A} A_{1} ; \quad A_{1} \rightarrow^{k_{d A}} ; \quad \rightarrow^{k_{B}} b_{B} B_{1} ; \quad B_{1} \rightarrow^{k_{d B}} .
$$

We assume that in compartment 2 initially $A$ and $B$ are not present, but they are transported over time from compartment 1. Moreover, in order to obtain simpler analytic expressions we assume $k_{d A}=k_{d B}$ and $b_{A}=b_{B}=b_{A B} \in \mathbb{N}$. That is, $A_{1}$ and $B_{1}$ appear in compartment 1 according to similarly noisy processes with average values respectively of $\frac{b_{A B} k_{A}}{k_{d A}}$ and $\frac{b_{A B} k_{B}}{k_{d A}}$. Under these assumptions analytic expressions for $F_{A_{2}}$ and $F_{B_{2}}$ can be derived and it is possible to show that $F_{A_{2}}$ and $F_{B_{2}}$ monotonically increase with $k_{1}$ and are bounded between the following limits, which are obtained by varying $k_{1}$ between 0 and infinity (as detailed in Supplemental Material [14] the bounds are obtained by solving the Lyapunov equation associated to the linear noise approximation of the chemical 


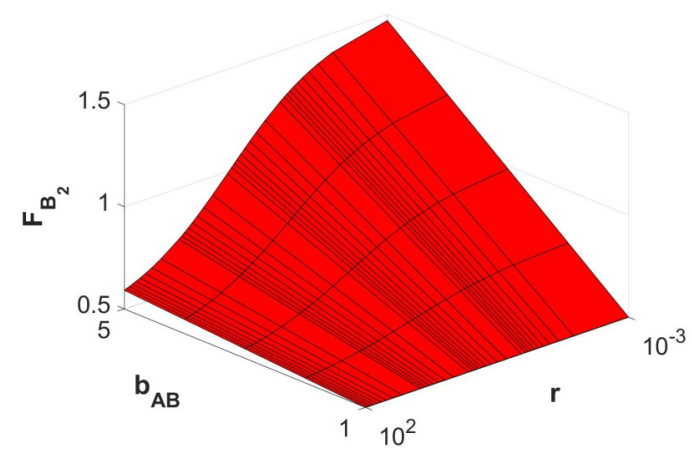

FIG. 2. Plot of upper bound of Fano factor of $B_{2}$ and $A_{2}$ at steady state as a function of the flux $r$ and of $b_{A B}$. Lower bound is identically 0.5 .

master equation (CME) [15]),

$$
\frac{1}{2} \leqslant F_{A_{2}} \leqslant \frac{k_{A}+b_{A B} k_{A}+4 \sqrt{k_{A} k_{B} r}+k_{B}+b_{A B} k_{B}}{4\left(k_{A}+2 \sqrt{k_{A} k_{B} r}+k_{B}\right)}
$$

and similarly for $F_{B_{2}}$.

Equation (7) implies that for $b_{A B}=1$ (Poisson noise), we always have

$$
F_{A_{2}}=F_{B_{2}}=\frac{1}{2},
$$

independently of the various parameters, while for $b_{A B}=2$, we have $F_{A_{2}}$ and $F_{B_{2}}$ bounded between $\frac{1}{2}$ and $\frac{3}{4}$, still significantly below Poisson levels.

As the Fano factor of $A_{2}$ and $B_{2}$ is monotonic in $k_{1}$, from Eq. (7) we also obtain that when $k_{1}$ is small enough, then the Fano factor of $A_{2}$ and $B_{2}$ will converge to $\frac{1}{2}$ independently of the various parameters. However, if $k_{1}$ is not small, then the noise will depend of both $b_{A B}$ (which represents the dimension of burts in the input) and $r$. The case when $r$ is small is the more interesting one, because it is where the reverse transport is slow, which is common in natural active transport mechanisms. In such a situation, input noise is always reduced to a Fano factor smaller than $\frac{1+b_{A B}}{4}$, requiring $b_{A B} \geqslant 4$ to exceed Poisson noise in the output. In Fig. 2 we plot the upper bound of $F_{A_{2}}$ and $F_{B_{2}}$ as a function of $r$ and $b_{A B}$ with the further assumption that $k_{A}=k_{B}$. As expected, when $b_{A B}=1$, the noise is always half of Poisson (Fano factor of $\frac{1}{2}$ ), instead for $b_{A B}>1$, the noise reduction depends on $r$, and always converges to half of Poisson for $r \rightarrow \infty$. Thus, preference toward the reverse direction of the symporter will always reduce noise to half of Poisson, while facilitated diffusion $(r=1)$ and active transport against the gradient can also lead to such reduction, in case the input noise is limited.

In the analysis above we assumed that $A_{2}$ and $B_{2}$ are produced with same rate. However, it could happen that $A_{2}$ and $B_{2}$ are produced with different rates because of a different chemical environment in the second compartment for each of the species. Nevertheless, as illustrated in detail in the Supplemental Material [14], the fact that $A_{2}$ and $B_{2}$ may be produced with different rates does not affect the noisereduction capability of a symporter model. Note also that in the previous analysis we assumed that $A_{1}$ and $B_{1}$ are affected by Poisson $\left(b_{A}=1\right)$ or super-Poisson $\left(b_{A}>1\right)$ noise and for this class of processes we always observe a lower bound on the noise of $\frac{1}{2}$. Nevertheless, as detailed in the Supplemental Material [14], if $A_{1}$ and $B_{1}$ are produced with sub-Poisson processes, then the noise is still reduced and with a Fano factor possibly smaller than $\frac{1}{2}$. Hence, this illustrates how symporter transport mechanisms can be effective in term of noise reduction even when the input species are already subPoisson.

Antiporters cotransport different molecule types in the opposite direction, picking up them at the two separate sides of the membrane and after a flip releasing on the other side. This can be modelled by the transport of molecules A and B between compartments 1 and 2 with the following reactions [Fig. 1(III)]:

$$
A_{1}+B_{2} \rightarrow^{k_{1}} A_{2}+B_{1} ; \quad A_{2}+B_{1} \rightarrow^{k_{1} \cdot r} A_{1}+B_{2} .
$$

Although the mechanism is different, at this level of description the reactions in (8) are identical to the ones in (5). Hence, under the same assumptions, the same analysis applies to this system, resulting in

$$
\frac{1}{2} \leqslant F_{A_{2}} \leqslant \frac{k_{A}+b_{A B} k_{A}+4 \sqrt{k_{A} k_{B} r}+k_{B}+b_{A B} k_{B}}{4\left(k_{A}+2 \sqrt{k_{A} k_{B} r}+k_{B}\right)}
$$

and similarly for $F_{B_{2}}$, where $b_{A B}=b_{A}=b_{B}$.

\section{Including degradation in the model}

In the previous analysis we implicitly assumed that the transport is much faster than any degradation, so that internal loss of molecules can be neglected. Although this is a reasonable assumption for many natural systems, the degradation of the species will influence the noise in the limit. Hence, it is important to explicitly include species degradation in the model in order to get a clear picture of the noisereduction capabilities of the mechanisms presented in the previous sections. Therefore, in what follows, we extend the symporter model in Fig. 1(II) by assuming that species A and $\mathrm{B}$ are degraded with the same rate $d$ both in compartment 1 and 2. Then, under the assumption that $\mathrm{A}$ and $\mathrm{B}$ appear in compartment 1 with Poisson processes with same mean $\frac{k_{A}}{k_{d A}}$, we obtain

$$
\begin{aligned}
& F_{A_{2}}=F_{B_{2}} \approx \frac{3}{4} \\
& F_{A_{1}}=F_{B_{1}} \approx 1+\frac{\sqrt{k_{A}^{2} r d^{2}}}{4 r k_{A}\left(d+k_{d A}\right)},
\end{aligned}
$$

which hold under the assumption that the transport rate is faster than all the other rates, as common in natural systems. Note that the Fano factor of $A_{2}$ and $B_{2}$ is always smaller than 1 (Poisson noise) for any combination of the parameters. However, if one considers the Fano factor of $A_{1}$ or $B_{1}$ at steady state, then this is always greater than 1 . Hence, this suggests that the symporter mechanism reduces the noise in one compartment by increasing the noise in the compartment where the species are produced. This is an important observation, because it implies that when a molecule is transported inside the cell from the external environment through a symporter membrane protein, then the molecules inside the cell will be less noisy than outside. 


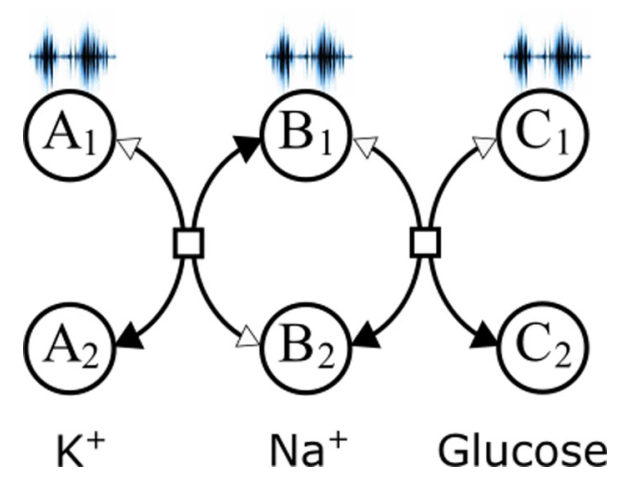

FIG. 3. Combination of an antiporter with a symporter. In this example we consider a sodium-potassium antiporter pump coupled with a glucose-sodium symporter [16]. We test with this how extracellular (top, molecules with subscript 1) noise in all three molecules could affect intracellular (bottom, molecules with subscript 2) signals downstream of these molecules.

Note that, as symporters and antiporters are symmetric, what we discussed in this section also holds for antiporters.

\section{COUPLED TRANSPORTERS}

Transporters work in combination with primary transporters such as the Na-K-ATPase antiporter establishing opposing gradients of sodium and potassium, through the expenditure of ATP. These gradients are then used as an energy source by secondary transporters to ferry other ions and molecules across the cell membrane. A simple interaction between primary and secondary transporters is depicted in (Fig. 3) where an antiporter (left) establishes a gradient in the $\mathrm{B}$ molecules that can be used by a symporter (right) to ferry $\mathrm{C}$ molecules inside the cell, and the relative reactions are modelled here below. We assume a noisy environment outside the cell (top) and we investigate the corresponding noise levels inside the cell (bottom).

$$
\begin{array}{ll}
A_{1}+B_{2} \rightarrow^{k_{1}} A_{2}+B_{1} ; & A_{2}+B_{1} \rightarrow^{k_{2}} A_{1}+B_{2} \\
B_{1}+C_{1} \rightarrow^{k_{3}} B_{2}+C_{2} ; & B_{2}+C_{2} \rightarrow^{k_{4}} B_{1}+C_{1} .
\end{array}
$$

In order to derive simple-enough analytic expressions, we need to introduce some assumptions. First, we assume all species outside the cell are affected by Poisson noise with expected values respectively of $p_{A}, p_{B}$, and $p_{C}$. Moreover, we fix the rates $k_{1}=2$ and $k_{2}=0.1$, whose ratio is taken from plausible biological conditions, assuming a fast export of sodium coupled to fast import of potassium [16]. Finally, we further assume $k_{3}=k_{4}=k$ meaning that the symporter works without a preferred direction and only uses the earlier established gradient of sodium to bring glucose into the cells. Under these assumptions we can obtain expressions for the Fano factors of the various species at steady state:

$$
F_{A_{2}}=\frac{p_{B}+10 p_{C}}{p_{B}+20 p_{C}} \quad F_{B_{2}}=\frac{p_{B}+40 p_{C}}{2\left(p_{B}+20 p_{C}\right)} \quad F_{C_{2}}=\frac{1}{2} .
$$

Interestingly, as in Eq. (7) and Eq. (9), the Fano factor of the intracellular $\mathrm{C}$ species is always $\frac{1}{2}$, independently of the reaction rates and the molecular levels of $\mathrm{A}, \mathrm{B}$, and $\mathrm{C}$ outside

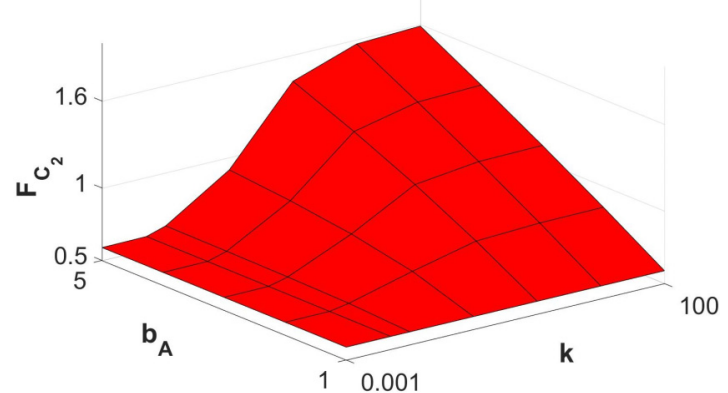

FIG. 4. Fano factor intracellular glucose. The figure plots the Fano factor of $C_{2}$ at steady state as a function of the rate of the transport $k=k_{3}=k_{4}$ and the bursts on the input process $b_{A}$. The figure has been obtained numerically by solving the LNA.

the cell. This shows how the results obtained when studying symporters and antiporters in isolation can still hold for more complex architectures. For A and B, instead, the Fano factors depend on the molecular levels of the various species outside the cell. For $A_{2}$, the noise is always sub-Poisson.

Beyond these analytic results, we can remove some of the simplifying assumptions by performing numerical simulations. In Fig. 3 we consider a more biologically realistic model (see Fig. 8 in the Supplemental Material [14] for a full description of the biological process), where we consider super Poisson noise outside the cell, a weaker reverse reaction for the symporter, and a stoichiometry for the antiporter matching a sodium-potassium pump [17]. That is, we replace the first two reactions in (10) with

$$
2 A_{1}+3 B_{2} \rightarrow^{2} 2 A_{2}+3 B_{1} \quad 2 A_{2}+3 B_{1} \rightarrow^{0.1} 2 A_{1}+3 B_{2} .
$$

We next use the linear noise approximation (LNA) [15,18,19] to numerically estimate the Fano factors. In Fig. 4 we plot the resulting Fano factor for the intracellular $\mathrm{C}$ species. It is possible to observe that, consistently with the analytic results, we still have that when $b_{A}=1$ (Poisson noise outside the cell), then $F_{C_{2}}$ is independent of $k$ and $\approx 0.6$. However, when $b_{A}>1$, then this system can still reduce the noise for any value of $k$. Nevertheless, the smaller the $k$ the more noise is filtered out.

\section{NUMERICAL ANALYSIS}

As detailed in the Supplemental Material [14], the bounds for symporters and antiporters are obtained by employing an approximation of the CME [15]. Hence, in order to further validate our results, we perform an analysis based on stochastic simulations obtained by means of the Gillespie algorithm [20].

In Fig. 5 we perform stochastic simulations of a symporter that transports molecules $\mathrm{A}$ and $\mathrm{B}$ from compartment 1 to compartment 2. In Fig. 5(I) we consider a model where $A_{1}$ and $B_{1}$ have super-Poisson noise. Same processes are considered also in Fig. 5(II), but we also include the degradation of the species in the model and consider a faster transport. As expected, in both cases, $A_{2}$ and $B_{2}$ have reduced variability compared to $A_{1}$ and $B_{1}$. 


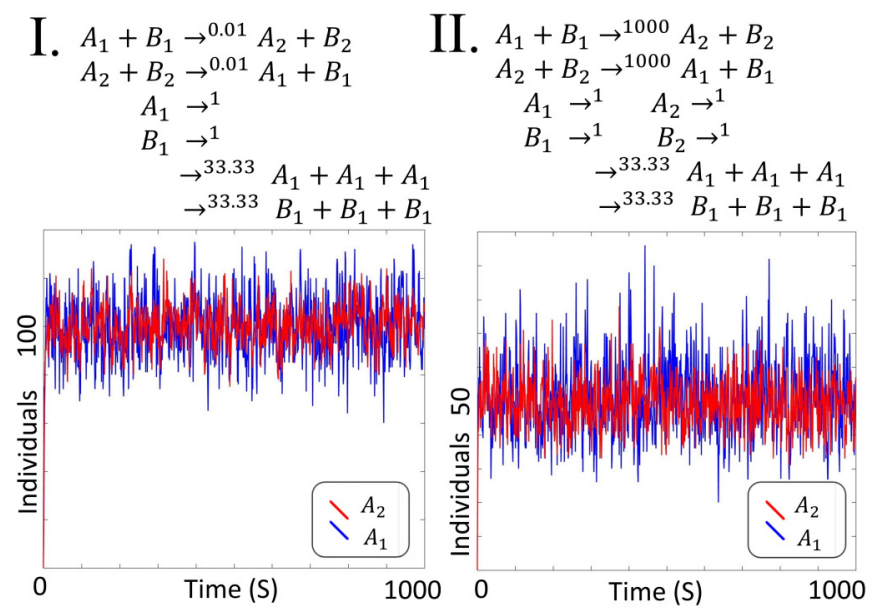

FIG. 5. Comparison of a model where the degradation of the species is assumed to be slower compared to all the other rates (I) and one where degradation of the species is explicitly considered (II). All plots are obtained by performing stochastic simulations of the CME [15]. In both cases it is possible to observe how $A_{2}$ has reduced variability.

In Fig. 6 we again consider a symporter transport with super-Poisson noise on the first compartment and we numerically estimate the time evolution of mean and variance. In Fig. 6(I) we consider the approximation of the CME we employed to derive the analytic expression in the main text. Then in Fig. 6 we numerically solve the CME.

$$
\begin{aligned}
& \text { I. } A_{1}+B_{1} \rightarrow^{0.01} A_{2}+B_{2} \\
& A_{2}+B_{2} \rightarrow^{0.01} A_{1}+B_{1} \\
& A_{1} \rightarrow^{1} \quad B_{1} \rightarrow^{1} \\
& \rightarrow^{2} A_{1}+A_{1}+A_{1} \\
& \rightarrow^{2} B_{1}+B_{1}+B_{1}
\end{aligned}
$$

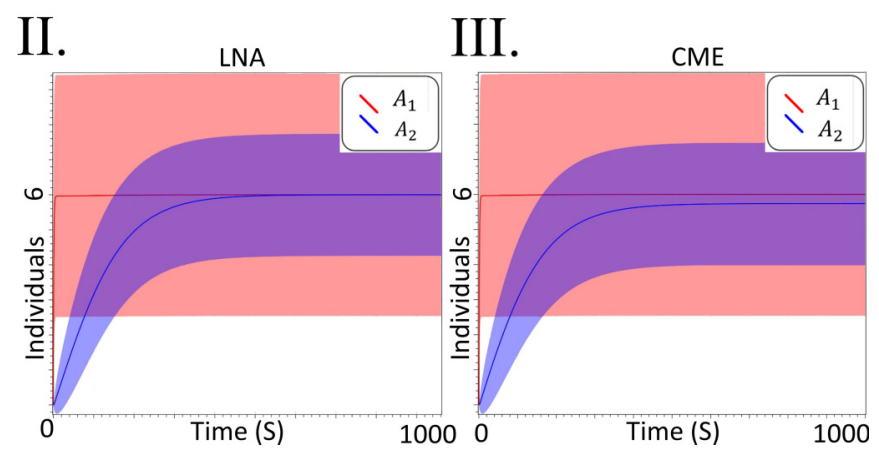

FIG. 6. We consider a reaction network where a symporter is used to transport molecules of species A and B between two compartments (I). In II we plot the time evolution of mean and variance $A_{1}$ and $A_{2}$ according to a linear noise approximation of the CME [15]. In III we plot the time evolution of the same species according to the CME. It is possible to observe that while the variance is equal in both figures, the mean is slightly different. This is due to the fact that the LNA only considers the first two moments of the distribution and neglects corrections terms of order higher than the first to estimate the mean. Note that this difference becomes less and less important the more molecules are in the system and is already negligible when $A_{1}$ and $B_{1}$ have a mean of few tens of molecules at steady state.

\section{DISCUSSION}

Various network motifs, such as feedback and feed-forward loops and annihilation filters, have been shown to reduce the noise in a homogeneous environment and the vast majority of related works focused on studying the noise suppression capabilities of these systems (see, e.g., Refs. [4,8,21]). In contrast, in this paper we show that spatial compartmentalization, and active mechanisms of transport in particular, are themselves efficient noise-reduction mechanisms often leading to sub-Poisson variability. Moreover, active mechanisms of transport can reduce noise without direct energy from ATP; in fact, these may also be passively driven by the concentration gradient of the molecules coupled by the transport reactions between compartments. Due to the ubiquitous nature of spatial compartmentalization in eukaryotic cells, this suggests that physical compartmentalization can be the dominant mechanism of noise reduction in eukaryotes. Network motifs can still be employed to further reduce the noise when particular precision is required. In fact, although symporters and antiporters naturally lead to sub-Poisson variability, these cannot reduce the noise to 0 , which instead can be achieved with certain nonlinear network motifs [4].

A key question is What features of symporters and antiporters are responsible for their effective noise-reducing capabilities? To investigate this issue we have tested subnetworks and found that the reversible release reaction of symporters and antiporters is an effective noise-reducing network motif in itself. For instance, if we consider a simple decomplexation situation given by the following reactions, where a complex $L$ reversibly releases his components, $A_{2}$ and $B_{2}$, at a given rate

$$
L \rightarrow^{k_{1}} A_{2}+B_{2} ; \quad A_{2}+B_{2} \rightarrow^{k_{2}} L
$$

where we assume that $L$ noise is modelled, for $b_{L}>0$, by

$$
\rightarrow^{k_{p}} b_{L} L ; \quad L \rightarrow^{k_{d}}
$$

then we can show that for any value of $k_{1}$ it holds that:

$$
\frac{1}{2} \leqslant F_{A_{2}}=F_{B_{2}} \leqslant \frac{\left(1+b_{L}\right) \sqrt{k_{d}}+4 \sqrt{b_{L} r k_{p}}}{4\left(\sqrt{k_{d}}+2 \sqrt{r b_{L} k_{p}}\right)}
$$

where $k_{2}=k_{1} r$ for $r \in \mathbb{R}_{\geqslant 0}$. This implies that for $b_{L}=1$ (Poisson input noise) we again have $F_{A_{2}}=F_{B_{2}}=\frac{1}{2}$ (half of Poisson independently of the rates). A similar pattern also occurs in the annihilation module proposed in Ref. [4] and a similar motif including a self-cleavage ribozyme has been shown to improve translational efficiency in Ref. [22]. Moreover, complex formation in homomers and heteromers has been shown to induce sub-Poisson variability in Ref. [23]. Thus, this suggests that a simple network motif combining complex formation and release steps may be a general motif for noise reduction.

In a biological example we have also focused on a system where a sodium-potassium antiporter pump creates a gradient of sodium that facilitates glucose import through a sodiumglucose symporter. Similar systems have been modelled 
by others [16], but here we show that this system can reduce the intracellular noise on glucose levels. Certainly this is just a small part of the glucose transport system as glucose can be imported in other ways [24] and it is also rather quickly processed into glucose 6-phosphate. Still, the mechanism proposed here could serve as a noise-reducing module ensuring that intracellular glucose signaling pathways are robustly controlled and do not give false signals for noise.

\section{ACKNOWLEDGMENT}

L.C. is partially funded by a Royal Society Professorship Grant No. RP120138.
[1] M. Kaern, T. C. Elston, W. J. Blake, and J. J. Collins, Stochasticity in gene expression: From theories to phenotypes, Nat. Rev. Gen. 6, 451 (2005).

[2] M. M. K. Hansen, R. V. Desai, M. L. Simpson, and L. S. Weinberger, Cytoplasmic amplification of transcriptional noise generates substantial cell-to-cell variability, Cell Syst. 7, 384 (2018).

[3] H. Kitano, Biological robustness, Nat. Rev. Genet. 5, 826 (2004).

[4] L. Laurenti, A. Csikasz-Nagy, M. Kwiatkowska, and L. Cardelli, Molecular filters for noise reduction, Biophys. J. 114, 3000 (2018).

[5] A. Becskei and L. Serrano, Engineering stability in gene networks by autoregulation, Nature 405, 590 (2000).

[6] M. Samoilov, A. Arkin, and J. Ross, Signal processing by simple chemical systems, J. Phys. Chem. A 106, 10205 (2002).

[7] A. Hilfinger, T. M. Norman, G. Vinnicombe, and J. Paulsson, Constraints on Fluctuations in Sparsely Characterized Biological Systems, Phys. Rev. Lett. 116, 058101 (2016).

[8] I. Lestas, G. Vinnicombe, and J. Paulsson, Fundamental limits on the suppression of molecular fluctuations, Nature 467, 174 (2010).

[9] T. Stoeger, N. Battich, and L. Pelkmans, Passive noise filtering by cellular compartmentalization, Cell 164, 1151 (2016).

[10] A. Singh and P. Bokes, Consequences of mrna transport on stochastic variability in protein levels, Biophys. J. 103, 1087 (2012).

[11] S. Smith and R. Grima, Single-cell variability in multicellular organisms, Nat. Commun. 9, 345 (2018).

[12] N. Battich, T. Stoeger, and L. Pelkmans, Control of transcript variability in single mammalian cells, Cell 163, 1596 (2015).
[13] C. A. Kaiser, M. Krieger, H. Lodish, and A. Berk, Molecular Cell Biology (WH Freeman, London, 2007).

[14] See Supplemental Material http://link.aps.org/supplemental/ 10.1103/PhysRevE.101.012414 for mathematical details, proofs, and additional examples.

[15] N. G. Van Kampen, Stochastic Processes in Physics and Chemistry, Vol. 1 (Elsevier, Amsterdam, 1992).

[16] K. Thorsen, T. Drengstig, and P. Ruoff, Transepithelial glucose transport and $\mathrm{na}+/ \mathrm{k}+$ homeostasis in enterocytes: An integrative model, Am. J. Physiol. Cell Physiol. 307, C320 (2014).

[17] E. M. Wright, D. D. F. Loo, and B. A. Hirayama, Biology of human sodium glucose transporters, Physiol. Rev. 91, 733 (2011).

[18] L. Cardelli, M. Kwiatkowska, and L. Laurenti, Stochastic analysis of chemical reaction networks using linear noise approximation, Biosystems 149, 26 (2016).

[19] R. Grima, Linear-noise approximation and the chemical master equation agree up to second-order moments for a class of chemical systems, Phys. Rev. E 92, 042124 (2015).

[20] D. T. Gillespie, Exact stochastic simulation of coupled chemical reactions, J. Phys. Chem. 81, 2340 (1977).

[21] J. M. Schmiedel, S. L. Klemm, Y. Zheng, A. Sahay, N. Blüthgen, Debora S. Marks, and Alexander van Oudenaarden, Microrna control of protein expression noise, Science 348, 128 (2015).

[22] N. Delalez, A. Sootla, G. H. Wadhams, and A. Papachristodoulou, Design of a synthetic srna-based feedback filter module, BioRxiv 504449 (2018).

[23] Z. Xu, K. R. Ghusinga, and A. Singh, Noise analysis in biochemical complex formation, BioRxiv 310847 (2018).

[24] I. S. Wood and P. Trayhurn, Glucose transporters (glut and sglt): Expanded families of sugar transport proteins, Br. J. Nutr. 89, 3 (2003). 